\title{
EPL \\ IN AN EVENTFUL ENVIRONMENT
}

I Bart van Tiggelen - DoI: https://doi.org/10.1051/epn/2019303

- LPMMC - University Grenoble Alpes/CNRS - Grenoble, France

Europhysics Letters was cofounded in 1986 by 17 European learned societies, and merged the two existing Letter journals Lettere al Nuovo Cimento - published by the Società Italiana di Fisica (SIF) - and the Journal de Physique Lettres, from the Société Française de Physique (SFP). The original idea was to create a real European Letter journal competitive with Physical Review Letters of the American Physical Society. The major scientific force behind EPL is the European Physical Society (EPS) that celebrated its $50^{\text {th }}$ anniversary only last year in Geneva. The publication of Europhysics Letters, re-baptized EPL in 2007 to emphasize its global impact, is a joint venture of the publishing houses of three physical societies: the Institute of Physics (IOP), the SIF, and the SFP.
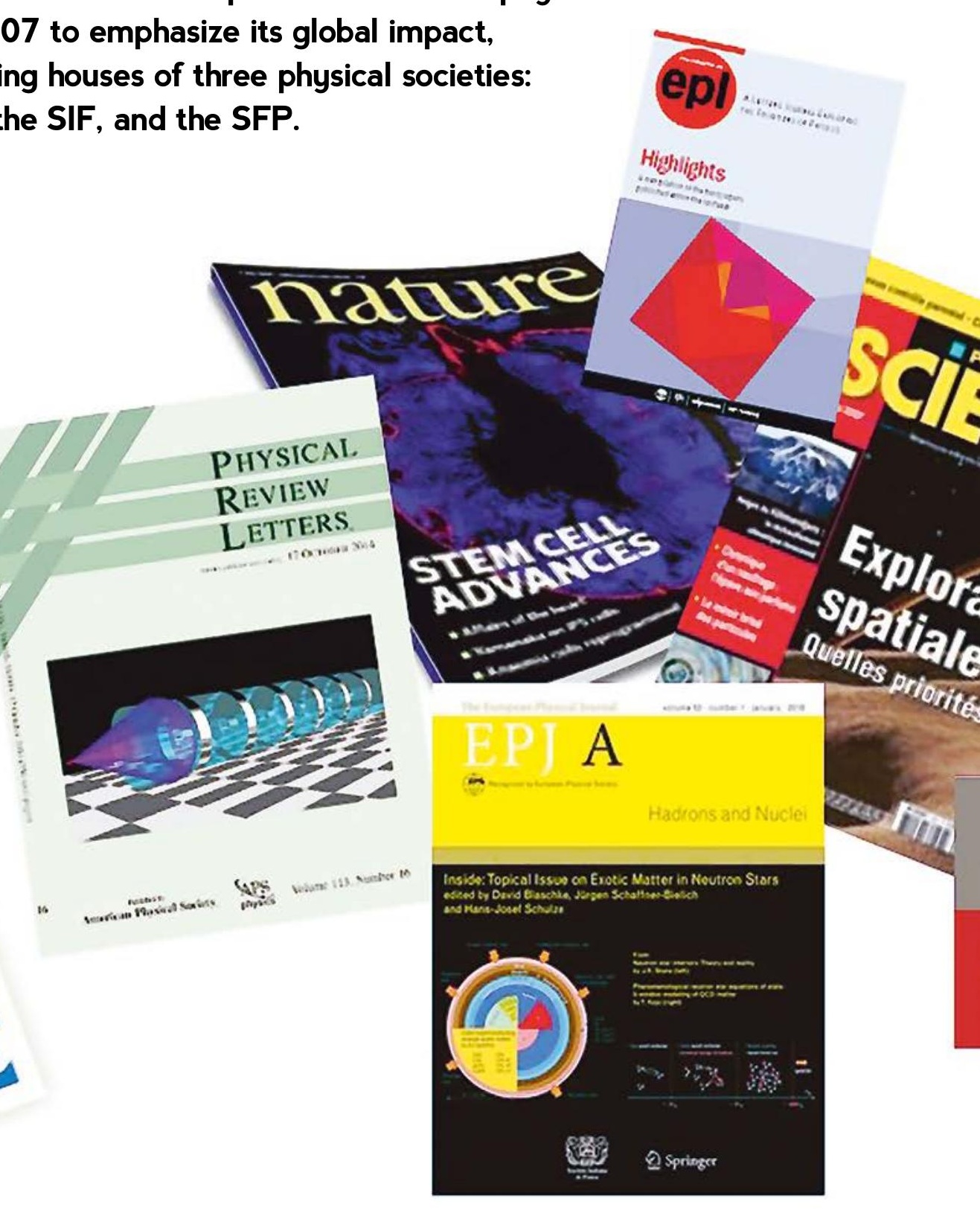
$T$ he launch of scientific journals by physical societies started more than one century ago "to give a new impulse to physics, to stimulate training, to excite the spirit of research, and to initiate discoveries". This is a quote attributed to Jean Perrin when the scientific publisher EDP Sciences was founded exactly one century ago by the SFP, supported by several distinguished scientists, such as Marie Curie and Louis de Broglie. The creation of physics journals started much earlier. The oldest two, Le Journal des Savants and the Philosophical Transaction, date back to 1665, a time where physics was still part of more general science, including medicine, biology, chemistry and even literature, published under the auspices of the national Scientific Academies, the Institut de France and the Royal Society respectively. The first commercial journals followed, such as the Philosophical Magazine in 1798 by Taylor \& Francis and Annalen der Physik in 1790, still published today by Wiley. Il Nuovo Cimento was probably one of the first journals founded by a pure physical society, created in 1855 to become the flagship journal of the SIF. Others followed rapidly, in 1873 the Russian Academy of Science founded the Journal of the Russian Society for Physics and

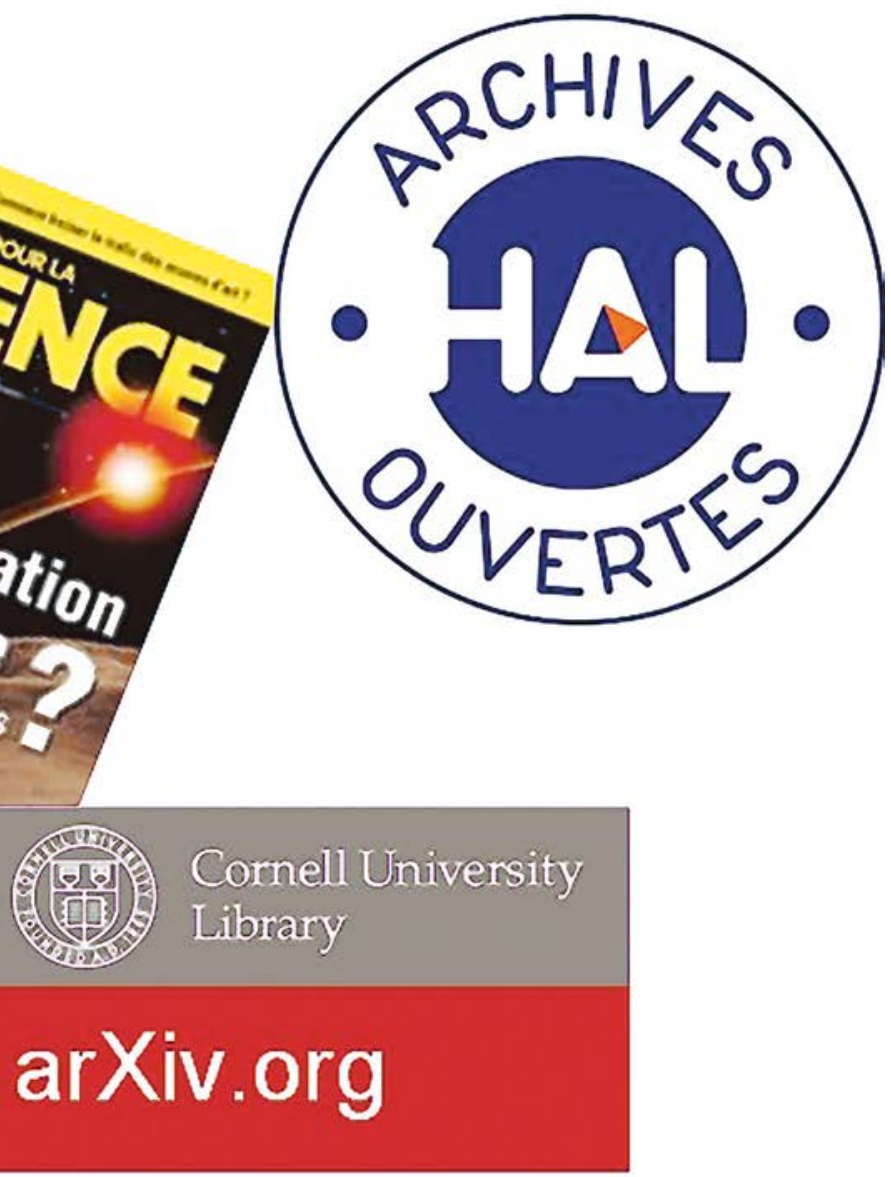

Chemistry, later re-baptized JETP. In 1874 the Journal of Physics was created by the IOP in the UK, the Physical Review by the American Physical Society in 1893.

The statement by Jean Perrin is still the reason why scholarly journals exist, but the landscape has changed dramatically after one century. The most significant change has been the arrival of Internet. Print versions have almost disappeared and articles can now be accessed in two clicks. Such easy access to all research sounds wonderful; it makes science move forward faster, and innovation is undoubtedly boosted when industry utilises scholarly articles. However, the commercial value of science has not gone unnoticed. Commercial publishers exist today that make profits in excess of $30 \%$, to disseminate research articles. A large scale study conducted in 2011 showed that the scientific publishing industry that year generated roughly 10 billion USD in revenue. With 2 million English language articles published in 2011 this is equivalent to roughly 3000 today's Euros for each article published worldwide. Journal prestige seems to have become a major tool for commercial exploitation. The economic model is simple but efficient: Accept only potentially high-impact articles that will raise the Impact Factor of the journal. This indicator, originally introduced by libraries as a tool to identify the journals to be purchased, counts the number of citations to all articles published in one year during the two years that follow. The higher the Impact Factor, the more attractive the journal becomes. Since rejecting papers costs money, the subscription fee for libraries also increases.

No need to insist that this model is highly unfair to the scientific community. Articles are rejected on their lack of direct impact, rather than on their "real" quality and originality, which we all know in physics often takes many years to reveal. Prestige is valuable to the community and clearly privileges researchers with prestigious grants. The threat of this vicious circle is that prestige gradually infiltrates the assessment and funding of research. In several European countries, such as Finland, the research budget of a laboratory or university depends explicitly on the number of articles published in journals with high Impact Factor. This unfortunate drift of science, is hardly a new impulse to physics. It is hardly exciting, and it hardly stimulates our students to "go where no one has gone before". Finally, it is by far the most inefficient way to favour blue-sky research, which for several centuries long has been the one and only trigger for important discoveries. Jean Perrin would have been disappointed.

Physics journals run by physical societies still exist and survive. For historic reasons, the landscape in Europe is more biodiverse than in the US, where the Physical Review journals have an almost monopoly position and attract many European scientists. In physics the most important learned society journals are the Journal of Physics series (IOPP), the New Journal of Physics, the 
full open-access journal in Europe founded by IOPP and DPG in 1998, and of course EPL, published by SIF, EDP Sciences, EPS, and IOPP. A few good journals are still published by national physical societies such as Acta Physica Polonica and Il Nuovo Cimento. Several others merged into the European Physical Journal platform EPJ

44

\section{It is crucial that physical societies keep taking the lead on scientific publishing; after all, they represent the physics community. It is important that they work together, without internal competition, and keep insisting on scientific quality and readability of research papers as the only criteria that count. 7 7}

created in 1992, such as Portugaliae Physica, Zeitschrift für Physik, Acta Physica Hungarica and Journal de Physique, federating no less than 25 European physical societies and published by SIF, EDP Sciences and the commercial publisher Springer-Verlag. On the commercial side, we find the large Freedom collection by Elsevier, that includes the Physics Letters Series, as well as Physics Reports, and the many journals published by the joint company Springer Nature created in 2015. The Springer journals focus on specialized communities such as fluid mechanics, statistical or mathematical physics. On the Nature side we find the prestigious, broad scope journals Nature, Nature Physics, and Scientific Reports, managed using the clever cascade model to keep rejected manuscripts in-house.

This huge European biodiversity makes the piece of the cake for each journal very small. It is crucial that physical societies keep taking the lead on scientific publishing; after all, they represent the physics community.
It is important that they work together, without internal competition, and keep insisting on scientific quality and readability of research papers as the only criteria that count. This gentle reminder was issued in 2012 in the form of the San Francisco Declaration on Research Assessment (DORA). An important role exists for the European Physical Society to coordinate these efforts in Europe. EPL is intended to be at the service of the whole community, with a broad scope and transparent rules, providing the professional support of the four editorial offices in Bologna, Bristol, Mulhouse and Paris, all run by physical societies. As is the case for most such journals, the benefits of the EPL Association flow back to their 17 European partner societies who benefit each year from a vital contribution to their tight annual budget. EPL also supports many poster prizes at international conferences, especially for young students. This also implies that physical societies should encourage their members to publish in "their" journals, like in the old days.

EPL and EPJ are two examples that demonstrate that by joining forces visibility and quality increase. My ultimate dream would be to create a European Platform comprising all learned society journals. Journals and publishers face a few major challenges in the near future, and the physical societies have to stand together if they do not want "others" to decide. The first is undoubtedly the reinvention of peer-review. All articles submitted to EPL get on average 1.8 reviews by expert peers, who evaluate the reported research on validity, broad interest, originality and readability. Getting independent reports within a reasonable time is hard work, since only $50 \%$ of the requests for review results in a report. Yet, more than $80 \%$ of all physicists recognize the importance of and need for an a posteriori quality check, and confirm that their paper improved after review. This was reported as the outcome of a survey conducted by Elsevier in 2009, and was confirmed by a similar survey conducted last year among members of the SFP.

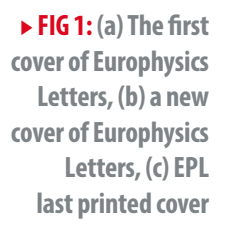

Volume 124

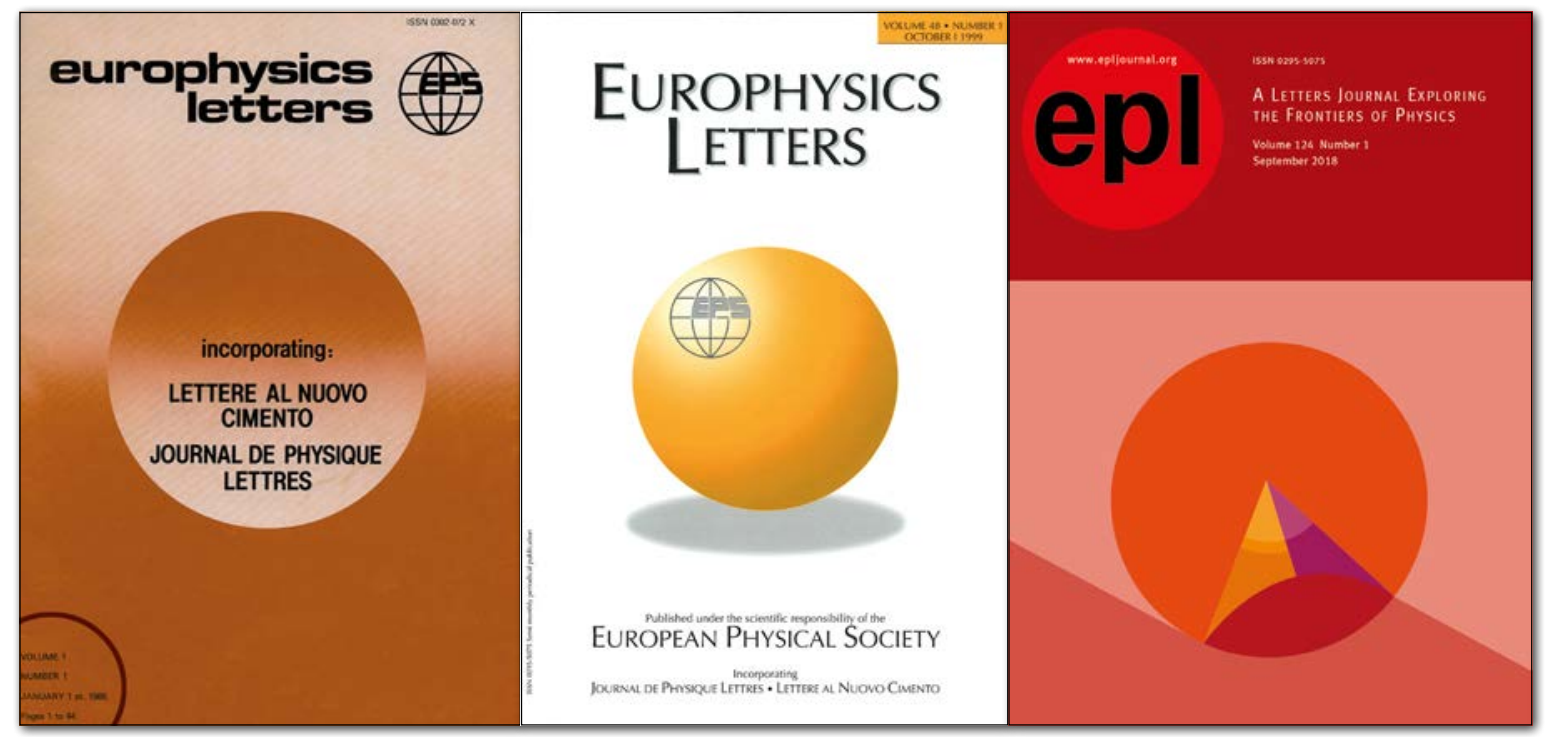


Nevertheless, the basic "peer review" principle, that all authors be reviewers and vice versa, suffers from broken symmetry. What can we do to make review attractive again? How do we get more recognition and visibility for the referees? Should we publish the report together with the article if the reviewer agrees? Should we create a worldwide database containing all active reviewers, updated by all journals?

The second challenge is Open Access. The arrival of Internet has made the subscription-based model obsolete and maybe perverse. The paper is online but impossible to access if you are connected outside the perimeter of your institution. The online access, that has replaced the shipping of print versions, has made libraries confronted with the obligation to accept "Big Deals", a popular term that refers to huge packages of journals, rather than to choose their own catalogue $\dot{a}$ la carte. Subscription fees grow faster than inflation rates, and become impossible to support for less-developed countries. Finally, the transfer of copyright to publishers has been bothering both authors and their employers for a while but is necessary because the publisher cannot sell what it does not own. The Internet facilitates easy access and rapid text mining but the current economic model prevents it. I am convinced that scientific publishing has a price and that scholarly journals should continue to exist if we want the scientists to do science, and not to spend their precious time looking out for referees, for proofreading, for indexing, and for publishing the articles on the net. It is the economic model that needs to be updated, and not the scientist. In the current subscription model, the reader or library pays the access. In an "author-pay" model the authors pay upfront for the publishing service and remain owner of their article. All readers would then be able to have Open Access "for free", and this will solve many issues raised earlier, including the (in most countries) ill-defined, so-called "Green" coexistence of preliminary versions on preprint servers and published versions in peer-reviewed journals under embargo. Physicists have a long tradition to distribute, deposit or self-archive preprints prior to publication. In an "author-pay" model, all versions can converge to one final version if the author wants this to happen.

Of course, the "author-pay" model is not the only route towards Open Access. A rich "biblio-diverse" landscape starts developing, where also institutional archives such as ORBi at Luik University in Belgium and HAL at CNRS and CEA in France start developing green policies on open access. The Green model is arguably not a model from the economic point of view, since the role of the publisher does not necessarily change. It is much more a highly justified cry for having no embargo on research articles produced by the employees of these institutions. The Gold "author-pay" breaks with the subscription model, but many colleagues protest against it, saying that "publishing should be for free", that "they do not want to be bothered by paying fees to journals", and that "they have no money to spend anyway". What they seem to ignore is that in the "reader-pay" model, our employers, or at the end of the day all tax-payers in the country, always have paid thousands of invisible Euros for the publication of their papers. In a successful implementation of any Open Access model tax-payers are again supposed to pay. Research organizations and universities in Europe have to stand together to negotiate agreements with the Publishing houses. Governments have to redirect the existing subscription fees to a unique national open-access platform. Quite recently, the cOAlitionS, a group of 15 European funding agencies endorsed by the European Commission, published their "Plan S" to force an Open Access transition. This initiative, that comes with a handful of recommendations and an ambitious timeline, will undoubtedly evolve in time. Most learned societies tend to support the open access initiative, but also realize that their journals, including EPL, cannot change the one-century-old economic model from one day to the other.

The publication of scientific articles must be carried out by professionals and thus comes with a price. However, the present economic model has become obsolete and perverse. Finding an international state-of-the-art ecosystem that responds to the needs of society and science requires an international force. National physical societies, with their century-old experience in publishing, have common interest and common knowledge. They should collaborate, control, and be proactive.

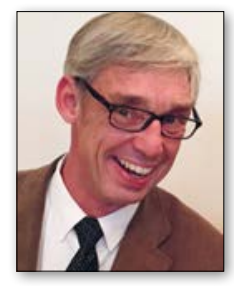

\section{About the Author}

Bart van Tiggelen is a research professor with CNRS in France, affiliated to LPMMC (University Grenoble Alpes/ CNRS) in Grenoble. In 2018 he was appointed Editor-in-Chief of EPL.

\section{Acknowledgment}

I would like to thank Emma Watkins (IOPP) and Agnès Henri (EDP Sciences) for their precious comments. 